\title{
Cutaneous Granuloma Revealing Familial Mercury Intoxication
}

\author{
Imène Rachdi ${ }^{1 *}$, Fatma Daoud ${ }^{1}$, Mayssam Jeridi ${ }^{1}$, Mehdi Somai ${ }^{1}$, Hana Zoubeidi ${ }^{1}$, Besma Ben Dhaou ${ }^{1}$, \\ Zohra Aydi ${ }^{1}$, Mohamed Hammami ${ }^{2}$ and Fatma Boussema ${ }^{1}$
}

${ }^{1}$ Faculty of Medicine, Department of Internal Medicine, Habib Thameur Hospital, University of Tunis El Manar, Tunisia

${ }^{2}$ National Institute for Research and Chemical and Physical Analysis, Tunisia

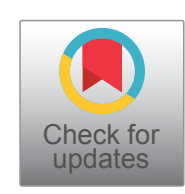

\begin{abstract}
Human exposure to mercury is known to lead to major health problems. Mercury toxicity effects multiple organs especially the central nervous system and kidneys. Skin diseases resulting from mercury exposure are various and may reveal intoxications. We report the case of a 60 -year-old male who had skin lesions that histologically showed foreign-body reaction with granuloma. The foreign body turned to be mercury. That unveiled paucal symptomatic family intoxication. Blood and urine analysis were run and found heavy metals including mercury surpassing normal range. The source of exposure remained unknown as the patient denied professional exposure or manipulation of inorganic mercury. The family was treated with chelation therapy over seas.
\end{abstract}

Keywords

Granuloma, Skin biopsy, Mercury, Intoxication

\section{Introduction}

Mercury is a heavy metal that can be found in different forms: Organic and inorganic. Human exposure to mercury is known to lead to major health problems. Symptoms of toxicity vary from a large panel of nonspecific signs like fatigue, dizziness to serious central nervous system impairment, renal failure and different skin conditions depending on the form of exposure [1]. We report the case of a 60 -year-old man in whom mercury toxicity was discovered as mercury was found in skin biopsies.

\section{Case report}

A 60-year-old man with a medical history of diabetes and rheumatoid arthritis was admitted into the internal medicine ward for arthralgia, the history taking revealed that he had rheumatoid arthritis since 2012 treated with methotrexate at a dosage of $20 \mathrm{mg}$ per week. In 2013, he presented with skin colorednodules on the abdomen and a white infiltrated plaque on the left elbow. These lesions were at first taken for rheumatoid or sarcoidnodules. Biopsies were performed. Histologically, the tissues demon strate dhistiocytic granulomas with giant cells and in flammatory in filtrate of eosinophils containing a purulent materiel, associated with fibrosis and necrosis. The purulent materiel turned to be mercury. In 2016, urine analysis showed the presence of mercury at the rate of $49.499 \mathrm{~m} \mathrm{cg} / \mathrm{g}$ creatinine, it also showed levels above normal of cadmium, lead, arsenic, copper and manganese. During the current stay, the patient showed no symptoms of neurological impairment. He just complained of chronic dizziness and fatigue. The physical examination found dental amalgam. Blood analysis at this point showed normal renal and hepatic functions as well as normal hemogram. We ran blood analysis searching for present mercury intoxication for the patient, his wife and his son and we found mercury at a rate of superior than the maximum contaminant level goals according to EPA (United States Environmental Protection Agency) [2]. Water analysis for mercury was done too, showing results under $2 \mathrm{ppb}$ in different water sources. The patient denied professional exposure to mercury, manipulation of thermometers, pesticides, skin mercury injection, also he didn't have a sea food rich diet. The patient and his wife claimed family intoxication of unknown source (since neither

*Corresponding author: Imène Rachdi, Faculty of Medicine, Department of Internal Medicine, Habib Thameur Hospital, University of Tunis El Manar, Tunis, Tunisia

Accepted: November 11, 2020

Published online: November 13, 2020

Citation: Rachdi I, Daoud F, Jeridi M, et al. (2020) Cutaneous Granuloma Revealing Familial Mercury Intoxication. J Healthcare 3(1):45-46 
the wife nor their son had amalgams and denied any sort of exposure to mercury). Back in 2016, the patient, his wife and his son had undergone chelation treatment.

\section{Discussion}

Elemental mercury can be found in thermometers, and dental amalgams. It is poorly absorbed from the gastrointestinal tract [3]. However, it can vaporize at room temperature and be absorbed through alveoli. It is usually responsible for acute mercury poisoning with central nervous system symptoms. Skin signs in acute inorganic mercury poisoning are rare butmay include acrodynia (pink disease), especially in children, and stomatitis [3]. Organic mercury is found as thimerosal, and sea food. Organic mercury poisoning is usually chronic and skin signs seem to be rare. Allergic contact dermatitis isthe most common form of mercury skin toxicity and can occur by both topical and systemic exposure [3], acrodynia results from chronic exposure to mercury. Cutaneous hyper pigmentation due to Mercurous chloride, mercurous oxide, and ammoniated mercury, often used topically for skin bleaching [4]. Cutaneous granuloma has been reported secondary to direct subcutaneous administration or extravasation of mercury injected into blood vessels, following metallic mercury injection, features of systemic mercury poisoning usually occur only when mercury enters the blood stream or lymphatics [5-8]. Treatment includes removing the source of mercury exposure and the use of chelating agents [3].

\section{Conclusion}

This case report presents an unusual form of cutaneous manifestation as mercury granuloma in a paucal symptomatic patient revealed a mercury toxicity in an entire family. The source of exposure has so far remainedunidentified.

\section{References}

1. Dantzig PI (2003) A new cutaneous sign of mercury poisoning? J Am Acad Dermatol 49: 1109-1111.

2. What are EPA's drinking water regulations for mercury? United States Environmental Protection Agency.

3. Bernhoft RA (2012) Mercury toxicity and treatment: A review of the literature. J Environ Public Health 2012: 460508.

4. Boyd AS, Seger D, Vannucci S, et al. (2000) Mercury exposure and cutaneous disease. J Am Acad Dermatol 43: 81-90.

5. Bradberry SM, Feldman MA, Braithwaite RA, et al. (1996) Elemental mercury-induced skin granuloma: $A$ case report and review of the literature. J Toxicol Clin Toxicol 34: 209-216.

6. Kayias EH, Drosos GI, Hapsas D, et al. (2003) Elemental mercury-induced subcutaneous granuloma. A case report and review of the literature. Send to Acta Orthop Belg 69: 280-284.

7. Altmeyer MD, Burgdorf MR, Newsome RE, et al. (2011) Cutaneous mercury granuloma: A case report Cutis 88: 189-193.

8. Bothale KA, Mahore SD, Pande S, et al. (2013) Cutaneous mercury granuloma. Indian J Occup Environ Med 17: 22-24.

DOI: $10.36959 / 569 / 461$ 